\title{
PATOLOGÍAS DE LOS DOCENTES DE LA PROVINCIA DE CORRIENTES Y EL IMPACTO EN LA ADMINISTRACIÓN DE RECURSOS DEL ESTADO
}

Amarilla, Alicia; Sanabria, Norberto; Corvalán, Rubén

\section{RESUMEN:}

Con este trabajo se pretende analizar las condiciones de trabajo en la que desarrollan su labor los docentes del nivel inicial y primario, cuales son las patologías que derivan de esta actividad y cómo impacta en los gastos del Estado. Además, presenta algunas propuestas que podrían colaborar, a través de políticas de estado, en mejorar las condiciones laborales de esta población.

PALABRAS CLAVES: docentes, licencias, salud, medicamentos, patologías.

\section{INTRODUCCION:}

La actividad laboral de los docentes representa un papel importante, tanto en la vida de la sociedad como en la partida presupuestaria que el Estado debe destinar a este sector para cubrir los haberes de los mismos, además del mantenimiento de establecimientos escolares, comedores de alumnos, inversión en capacitaciones profesionales, cubrir las vacantes ocasionadas por licencias del personal (las mismas pueden darse por razones de salud, cargo de mayor jerarquía, etc.).

La importancia de la actividad docente, la complejidad en la que desarrollan su actividad laboral, al ejercer su profesión frente a alumnos que oscilan entre los cuatro y catorce años de edad, las exigencias del sistema educativo con sus evaluaciones periódicas y actualización permanente, la diversidad de condiciones socio-culturales y económicas en que él trabaja este grupo a estudiar (niveles inicial y primario), lleva a pensar tanto, en su las condiciones de trabajo en las que desarrollan su labor, en su estado de salud actual y cuál sería el impacto en un servicio del Estado descentralizado de toda la provincia de Corrientes.

Por otra parte, conociendo la realidad actual, en la cual la obra social prestadora de servicios al personal de la administración pública provincial, en este caso especificando la población a estudiar "los docentes", ha detectado un alza en el consumo por parte de sus prestadores de medicamentos para tratamiento del destrés, ansiedad, depresión, entre otros. 
La labor docente se debe ajustar a un Calendario. Los Calendarios definen el tiempo de trabajo para, para los recursos individuales y para las tareas.

El Estado tiene "costos fijos" para el pago de la labor docente, a lo que se suman "otros costos" (consultas y/o estudios médicos, fármacos, etc).

Costo fijo: es una determinada cantidad monetaria presupuestada para una tarea. Estos costos son los mismos independientemente del tiempo o esfuerzo que los recursos empleen en la finalización de la tarea.

Por lo expuesto anteriormente, y porque no se disponía de antecedentes de investigación en estas temáticas para esta población (provincia de Corrientes), se exploró desde la siguiente perspectiva:

Motiva el diseño y aplicación de politicas que, desde el Estado, mejoren la condición laboral de los docentes de la Provincia de Corrientes y reduzcan los otros costos y costos ocultos.

Tema: Licencias por razones de Salud de los docentes estatales de nivel inicial y primario de la provincia de Corrientes y su relación con las patologías prevalecientes. Impacto en la administración de recursos del Estado.

\section{Objetivos:}

\section{General:}

Describir y cuantificar las patologías que conllevan al usufructo de licencias por razones de enfermedad de los docentes del nivel inicial y primario de la provincia de Corrientes, los medicamentos prescriptos a éste grupo de trabajadores y su impacto en el presupuesto del Estado.

\section{Específicos:}

A) Identificar las patologías prevalecientes de los docentes del sector público del nivel inicial y primario de la Provincia de Corrientes, dependientes del Consejo General de Educación (CGE).

B) Identificar y categorizar las características socio-demográficas de los docentes.

C) Inferir a través de los medicamentos prescriptos y licencias de salud otorgadas en un periodo determinado, como afectan las mismas a la administración de recursos del Estado.

A.4.Situación problemática: Existe una relación entre los problemas de salud, ciertas patologías y los usufructos de licencias de los docentes de la provincia de Corrientes. Ésta, en el caso de ser verificada, podría provocar al Estado un gasto extra para cubrir los puestos mediante Suplencias, mientras duren las licencias.

\section{A.5. Interrogantes:}

I. ¿Cuáles son las patologías presentes en los docentes del nivel inicial y primario?

II. ¿Estas patologías, provocan el uso de licencias de los docentes?

III. ¿Cuáles son los medicamentos más recetados a los docentes, y para qué casos de patologías se prescriben teniendo en cuenta la Acción y las Indicaciones medicamentosas de los mismos? 
IV. ¿Cómo afecta al Estado el uso de licencias del personal docente?

V. ¿Se puede realizar una propuesta al Estado para mejorar las condiciones del trabajo docente, reducir el número de solicitudes de licencias por enfermedad y disminuir el costo oculto que esto le ocasiona?

\section{METODOLOGÍA}

Este trabajo se focalizó en estudio de carácter cuali-cuantitativo, de tipo exploratorio y descriptivo.

Para el logro de estos objetivos se definieron una serie de variables a fin de abordar la situación problemática de los docentes estatales del nivel inicial y primario de la provincia de Corrientes, sobre las licencias, patologías e impacto en el Estado.

El Universo se conformó por los docentes dependientes del MEC.La Población, por la totalidad de los docentes del nivel inicial y primario dependientes del CGE de la provincia de Corrientes y laUnidad de análisisfue construido por los Docentes del Nivel Inicial y Primario, dependientes del CGE de la Provincia de Corrientes, que han usufructuado Licencias de Salud durante el año 2015, registradas en la Junta de Reconocimientos Médicos de la provincia (JRM).

Los Instrumentos de relevamientos de datos fueron como fuente primaria los informes cuantitativos suministrados por las autoridades de la JRM, el MEC, el IOSCOR. Como fuente secundaria se participó de reuniones con socios estratégicos, quienes brindaron datos cualitativos en base a su experiencia y función que desempeñan.
El análisis de los datos cuantitativos permitió la verificación de quienes y cuantos hacen uso de licencias de Salud, separados en 21 patologías de acuerdo al Sistema fisiológico del cuerpo humano.

Las charlas y reuniones con los informantes claves permitieron obtener datos cualitativos e información sobre la problemática planteada desde la perspectiva del Estado e identificar algunas de las condiciones de trabajo y el estado de salud en general.

La Estrategia de análisis utilizada fue la exploración de los datos cuantitativos, la segmentación mediante cuadros de valores numéricos y porcentuales de grupos, representación gráfica de barras y de pastel, utilización de estadística descriptiva a fin de obtener conclusiones mediante la estadística inferencial (Ross, S. 2000). Para la fuente secundaria se procedió a realizar preguntas abiertas, en la cual los informantes calificados aportaron información concisa, registradas en cuadros, textos y presentaciones en formato digital y anotaciones manteniendo la objetividad y neutralidad, transcribiendo sin hacer valoración personal.

El análisis orientó a construir desde una perspectiva, la realidad de los docentes del Nivel Inicial y Primario de la provincia de Corrientes.

El cuadro siguiente expone las variables y dimensiones que fueron analizadas para la investigación: 


\begin{tabular}{|l|c|}
\hline \multicolumn{1}{|c|}{ VARIABLE } & \multicolumn{1}{c|}{ DIMENSION } \\
\hline \multirow{2}{*}{ A) Condiciones de trabajo } & Contenido de trabajo \\
\hline \multirow{3}{*}{$\begin{array}{l}\text { B) Estado de salud de los } \\
\text { docentes }\end{array}$} & Modo de gestión de la fuerza de trabajo \\
\hline & Licencias de Salud \\
\hline \multirow{3}{*}{ C) Datos socio-demográficas } & Patologias \\
\cline { 2 - 2 } & Sexo \\
\cline { 2 - 2 } & Edad \\
\hline
\end{tabular}

Cuadro №1: Variables

DESARROLLO-

Procesamiento de datos
D. 1- los datos cuantitativos, que a continuación se exponen las dimensiones del organismo en el cual prestan servicios nuestra población seleccionada:

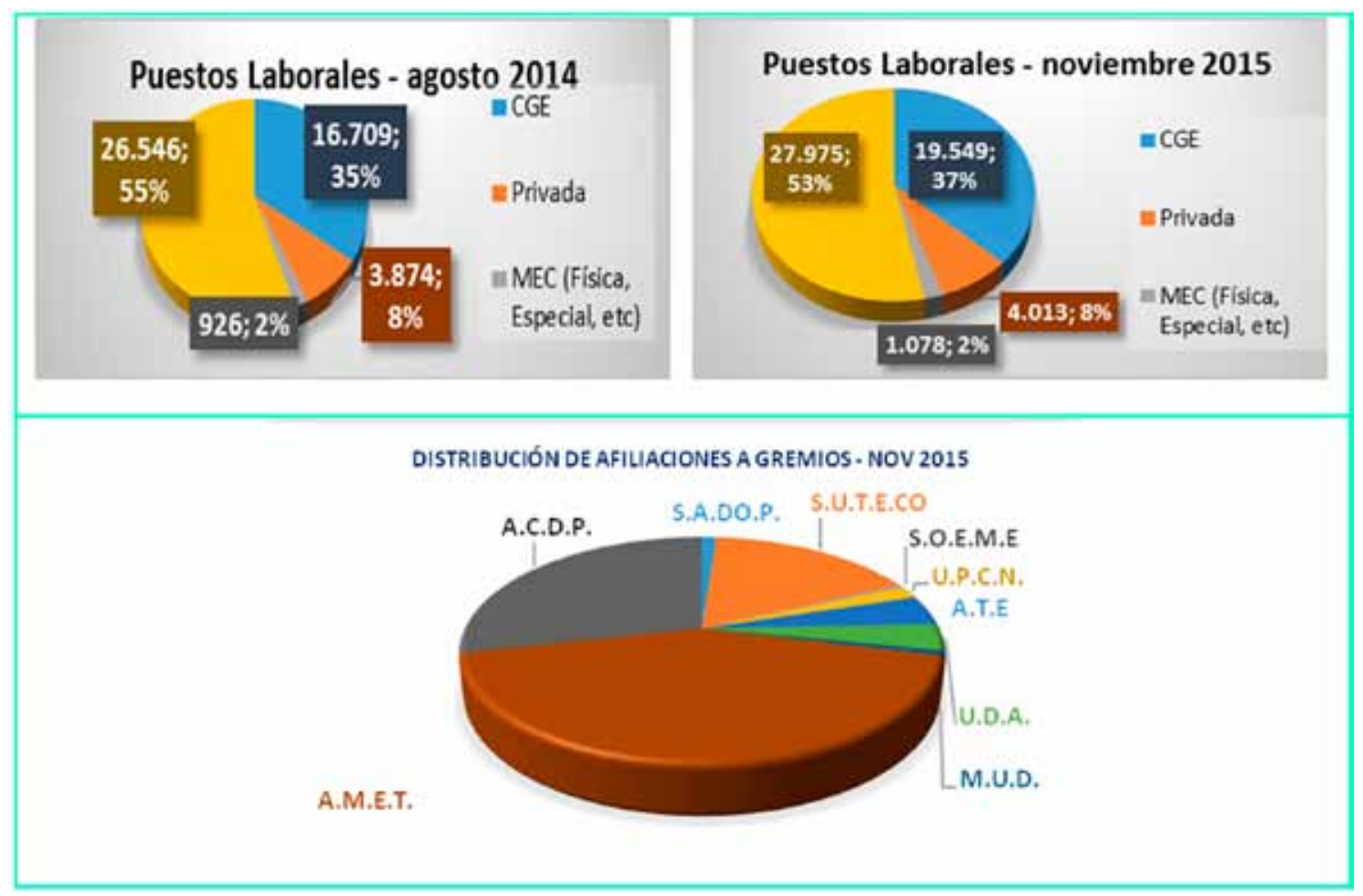

Gráfico No1: Puestos Laborales y Afiliaciones Sindicales Docentes (37\% de Agremiación) Agosto 2014 y Noviembre 2015 DRRHH-

[Datos del Departamento Estadistica e Información Educativa del MEC noviembre 2015] 


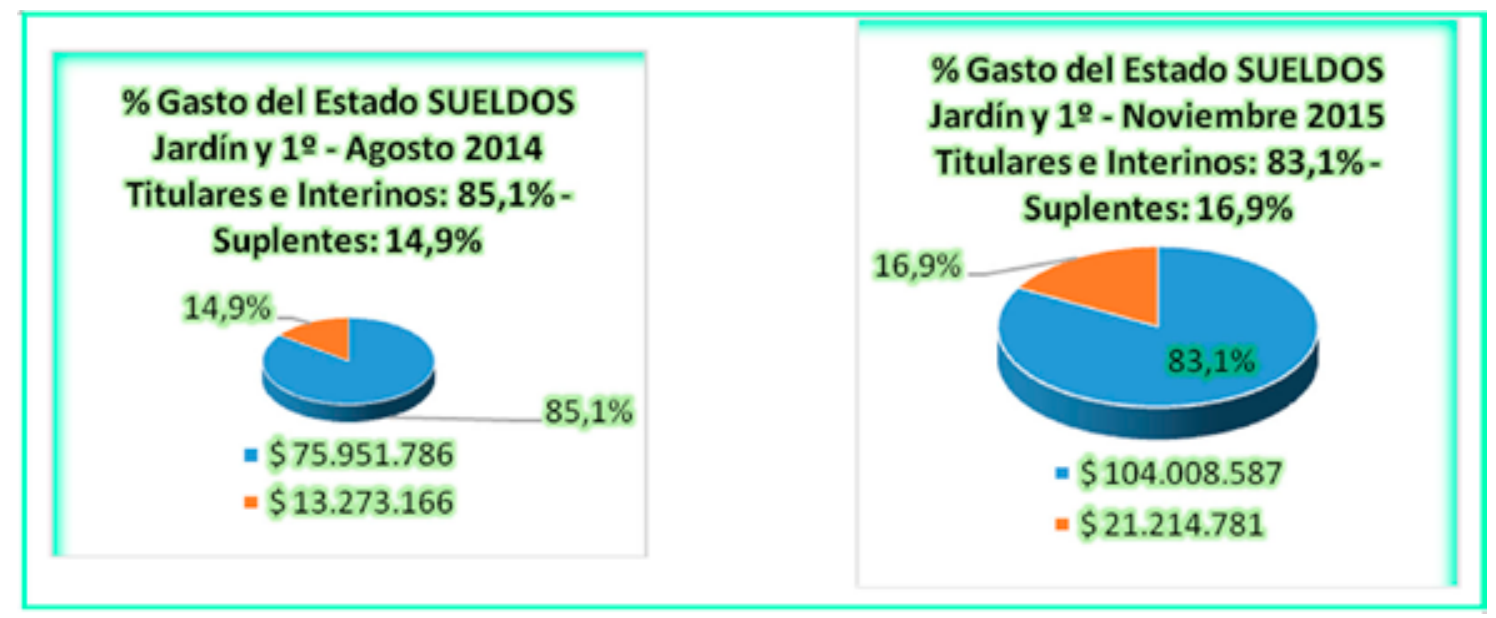

Gráfico No 2: Gasto Salarial del Estado en Maestros de Jardin y Primaria Agosto 2014 y Noviembre 2015
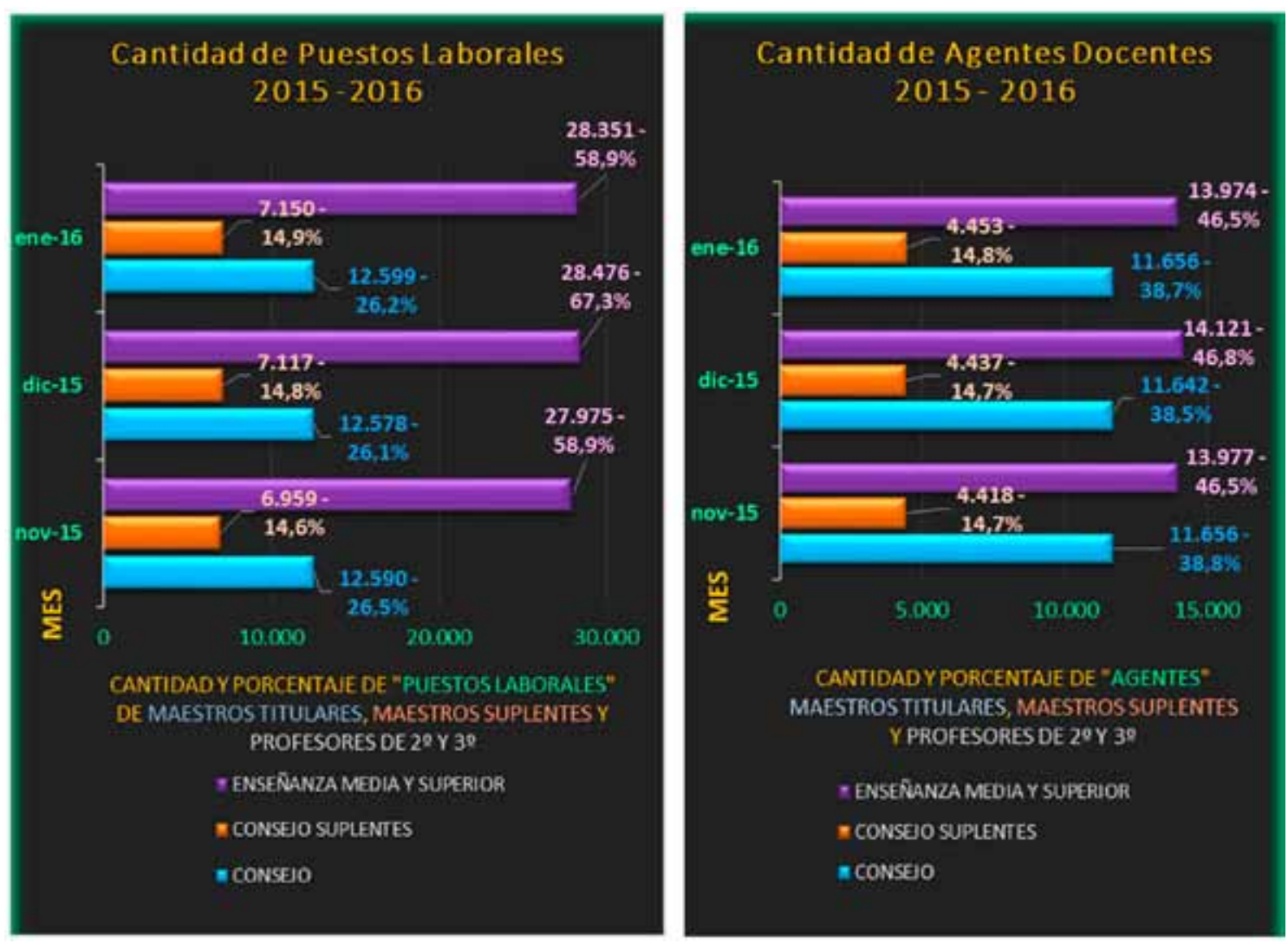

Gráfico No 3: Cantidad y \% de Puestos Laborales y Agentes Docentes de Jardín, 1a, $2^{a}$ y $3^{a}$ 

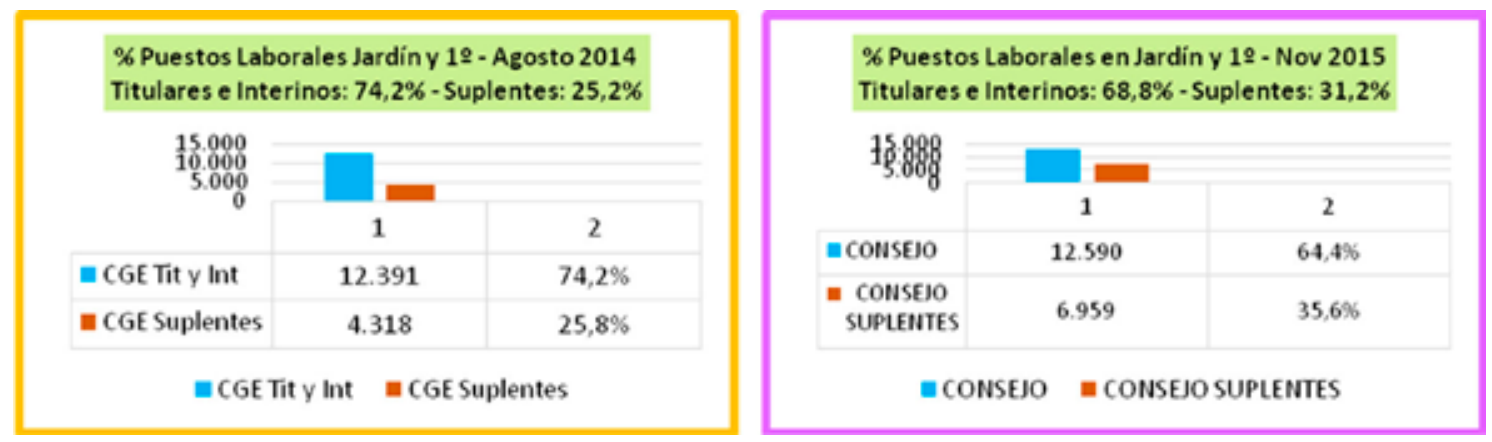

Gráfico No 4: Cantidad y \% de Puestos Laborales de Jardin y Primaria CGE - DRRHH

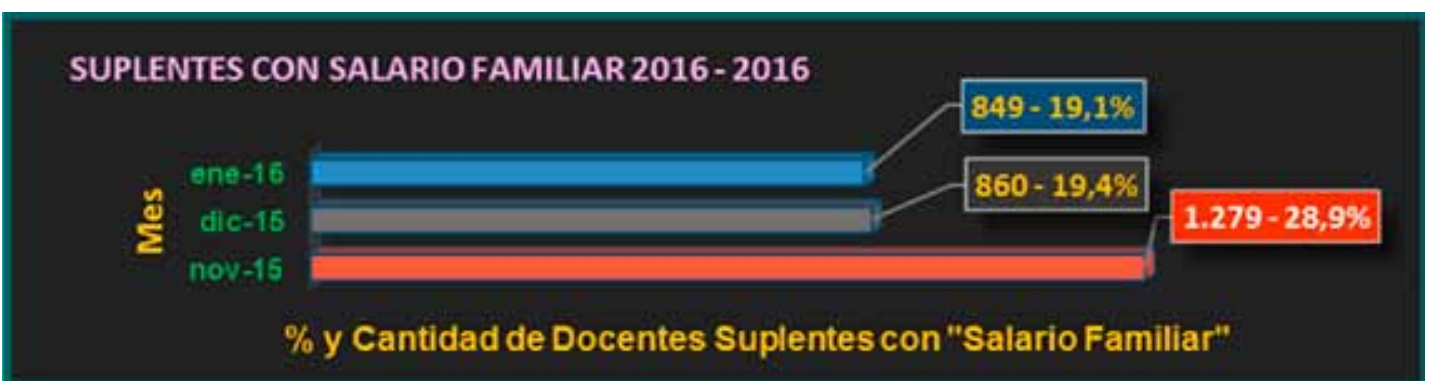

Gráfico No 5: Cantidad y \% de Maestros de Jardín y Primaria con Hijos - DRRHH-

Informantes Calificados: La información aportada por los Informantes Claves, permitió contextualizar la labor Docente en $\mathrm{Co}^{-}$ rrientes y el CONTENIDO DE TRABAJO de los mismo (VARIABLE estudiada).

- VARIABLE DATOS SOCIODEMOGRAFICOS Y CONDICIONES DE TRABAJO: A continuación se exponen di- versos cuadros con las VARIABLES seleccionadas,(MODODE GESTIÓN DE LA FUERZA DE TRABAJO, SEXO, ANTIGÜEDAD EN EL CARGO, EDAD, LUGAR DE TRABAJO, LICENCIAS, ETC.) con el objetivo de analizar, verificar y describir la situación de la población estudiada y permita llegar a conclusiones fehaciente de la realidad de los docentes:

\begin{tabular}{|c|c|c|}
\hline EDAD & CANTIDAD & $\%$ \\
\hline HASTA 25 & 288 & 0,77 \\
\hline $26-30$ & 1.976 & 5,29 \\
\hline $31-35$ & 3.979 & 10,65 \\
\hline $36-40$ & 5.038 & 13,48 \\
\hline $41-45$ & 6.243 & 16,71 \\
\hline $46-50$ & 7.136 & 19,10 \\
\hline $51-55$ & 6.458 & 17,28 \\
\hline $56-60$ & 4.112 & 11,01 \\
\hline 61 O MAS & 2.134 & 5,71 \\
\hline TOTAL & $\mathbf{3 7 . 3 6 4}$ & $\mathbf{1 0 0 , 0 0}$ \\
\hline
\end{tabular}

\begin{tabular}{|c|c|c|}
\hline ANTIGÜEDAD & CANTIDAD & $\%$ \\
\hline HASTA 5 AÑOS & 12.500 & 33,45 \\
\hline 6 A 10 & 8.501 & 22,75 \\
\hline 11 A 15 & 4.125 & 11,04 \\
\hline 16 A 20 & 4.453 & 11,92 \\
\hline 21 A 25 & 4.374 & 11,71 \\
\hline 26 A 30 & 2.450 & 6,56 \\
\hline MAS DE 30 & 961 & 2,57 \\
\hline TOTAL & 37.364 & 100,00 \\
\hline
\end{tabular}

Total Dias

Licencias

Docentes 2015

459.704

Cuadro No 2: Total de Licencias Docentes 2015, separadas por Edad y Antigüedad JRM 


\begin{tabular}{|c|c|c|}
\hline SEXO CGE & CANTIDAD & $\%$ \\
\hline HOMBRES & 1.683 & 10,11 \\
\hline MUJERES & 14.962 & 89,89 \\
\hline TOTAL & $\mathbf{1 6 . 6 4 5}$ & 100,00 \\
\hline
\end{tabular}

A

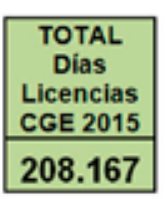

B

\begin{tabular}{|c|c|c|}
\hline $\begin{array}{c}\text { SEXO } \\
3^{\circ}\end{array}$ & CANTIDAD & $\%$ \\
\hline HOMBRES & 3.923 & 21,90 \\
\hline MUJERES & 13.994 & 78,10 \\
\hline TOTAL & 17.917 & 100,00 \\
\hline
\end{tabular}

C

Cuadro No 3: Licencias Docentes 2015, separadas por Sexo y Lugar $(A, C)$

\begin{tabular}{|c|c|c|c|c|c|c|c|c|}
\hline LUGAR & CANTIDAD & $\%$ & \multirow{2}{*}{ LUGAR } & \multirow{2}{*}{ CANTIDAD } & \multirow{2}{*}{$\%$} & \multirow{2}{*}{$\begin{array}{l}\text { SEXO- } \\
\text { Total } \\
\text { Licencias }\end{array}$} & \multirow[b]{2}{*}{ CANTIDAD } & \multirow[b]{2}{*}{$\%$} \\
\hline CONSEJO & 12.496 & $\begin{array}{c}33,4 \\
6\end{array}$ & & & & & & \\
\hline $\begin{array}{l}\text { CONSEJO- } \\
\text { SUPL }\end{array}$ & 4.149 & $\begin{array}{c}11,1 \\
1 \\
\end{array}$ & $\begin{array}{l}\text { CONSEJO -Tit, } \\
\text { Int y Suplentes }\end{array}$ & 16.645 & $\begin{array}{c}44,5 \\
7\end{array}$ & HOMBRES & 6.077 & $\begin{array}{c}16,2 \\
7\end{array}$ \\
\hline PRIVADA & 2.208 & 5,91 & \multirow{2}{*}{$\begin{array}{l}\text { PRIVADA - Tit, } \\
\text { Int, Suplentes }\end{array}$} & \multirow[b]{2}{*}{2.367} & \multirow[b]{2}{*}{6,34} & \multirow[b]{2}{*}{ MUJERES } & \multirow[b]{2}{*}{31.269} & \multirow[b]{2}{*}{$\begin{array}{c}83,7 \\
3\end{array}$} \\
\hline $\begin{array}{l}\text { PRIVADA - } \\
\text { SUPL }\end{array}$ & 159 & 0,43 & & & & & & \\
\hline MEDIA Y SUP & 15.143 & $\begin{array}{c}40,5 \\
5\end{array}$ & $\begin{array}{l}\text { MEDIA Y SUP - } \\
\text { Tit, In, Suplentes }\end{array}$ & 17.917 & $\begin{array}{c}47,9 \\
8\end{array}$ & TOTAL & 37.346 & 100,00 \\
\hline $\begin{array}{c}\text { MEDIA Y SUP - } \\
\text { SUPL }\end{array}$ & 2.774 & 7,43 & \multirow{2}{*}{$\begin{array}{l}\text { MINISTERIO - } \\
\text { Int, Suplentes }\end{array}$} & \multirow{2}{*}{417} & \multirow{2}{*}{1,12} & & & \\
\hline MINISTERIO & 408 & 1,09 & & & & & & \\
\hline $\begin{array}{l}\text { MINISTERIO - } \\
\text { SUPL }\end{array}$ & 9 & 0,02 & \multirow{2}{*}{ TOTAL } & \multirow{2}{*}{37.346} & \multirow{2}{*}{100,00} & & & \\
\hline TOTAL & 37.346 & 100,00 & & & & & & \\
\hline
\end{tabular}

Cuadro No 4: Total de Licencias Docentes 2015, separadas por Lugar y Condición Laboral y Sexo JRM

VARIABLE ESTADO DE SALUD gías), provisto por elJRM de Ctes. 2015 DE LOS DOCENTES :

Total de Licencias Docentes: 37.364

- DIMENSION

LICENCIAS - Total de Días de Licencia: 459.704 OTORGADAS POR RAZONES DE SALUD.

Total de Licencias de Maestras Comunes de Jardín y PrimaEstán separadas en 21 grupos (patolo- ria: 16.645 Total de Días: 208.167

\begin{tabular}{|c|r|c|}
\hline Dias Lic CGE & Cantidad & $\%$ \\
\hline 1 a 5 & 10.929 & 65,66 \\
\hline 6 a 10 & 863 & 5,18 \\
\hline 11 a 15 & 684 & 4,11 \\
\hline 16 a 20 & 246 & 1,48 \\
\hline 21 a 25 & 54 & 0,32 \\
\hline 25 a 30 & 2.859 & 17,18 \\
\hline 31 a 35 & 124 & 0,74 \\
\hline 36 a 40 & 66 & 0,40 \\
\hline 41 a 45 & 144 & 0,87 \\
\hline 46 a 50 & 7 & 0,04 \\
\hline 51 a 55 & 7 & 0,04 \\
\hline 56 a 60 & 435 & 2,61 \\
\hline 61 a 65 & 8 & 0,05 \\
\hline 66 a 89 & 17 & 0,10 \\
\hline 90 & 30 & 0,18 \\
\hline 91 a 120 & 32 & 0,19 \\
\hline 121 a 150 & 107 & 0,64 \\
\hline 151 a 180 & 28 & 0,17 \\
\hline 181 a 300 & 5 & 0,03 \\
\hline TOTAL & 16.645 & 100,00 \\
\hline
\end{tabular}

\section{Mujeres Maestras CGE}

\begin{tabular}{|c|r|c|}
\hline EDAD & CANTIDAD & $\%$ \\
\hline 21 a 25 & 96 & 0,64 \\
\hline 26 a 30 & 658 & 4,40 \\
\hline 31 a 35 & 1.037 & 6,93 \\
\hline 36 a 40 & 1.815 & 12,13 \\
\hline 41 a 45 & 2.664 & 17,81 \\
\hline 46 a 50 & 3.603 & $\mathbf{2 4 , 0 8}$ \\
\hline 51 a 55 & 2.874 & 19,21 \\
\hline 56 o mas & 2.215 & 14,80 \\
\hline TOTAL & $\mathbf{1 4 . 9 6 2}$ & 100,00 \\
\hline
\end{tabular}

Cuadro No 5: Total de Licencias Docentes, separado según el sexo

\section{Hombres Maestros CGE}

\begin{tabular}{|c|r|c|}
\hline EDAD & CANTIDAD & $\%$ \\
\hline 21 a 25 & 7 & 0,42 \\
\hline 26 a 30 & 71 & 4,22 \\
\hline 31 a 35 & 166 & 9,86 \\
\hline 36 a 40 & 178 & 10,58 \\
\hline 41 a 45 & 299 & 17,77 \\
\hline 46 a 50 & 383 & 22,76 \\
\hline 51 a 55 & 259 & 15,39 \\
\hline 56 a 60 & 248 & 14,74 \\
\hline 61 o mas & 72 & 4,28 \\
\hline TOTAL & 1.683 & 100,00 \\
\hline \multicolumn{3}{|c}{} \\
\hline
\end{tabular}




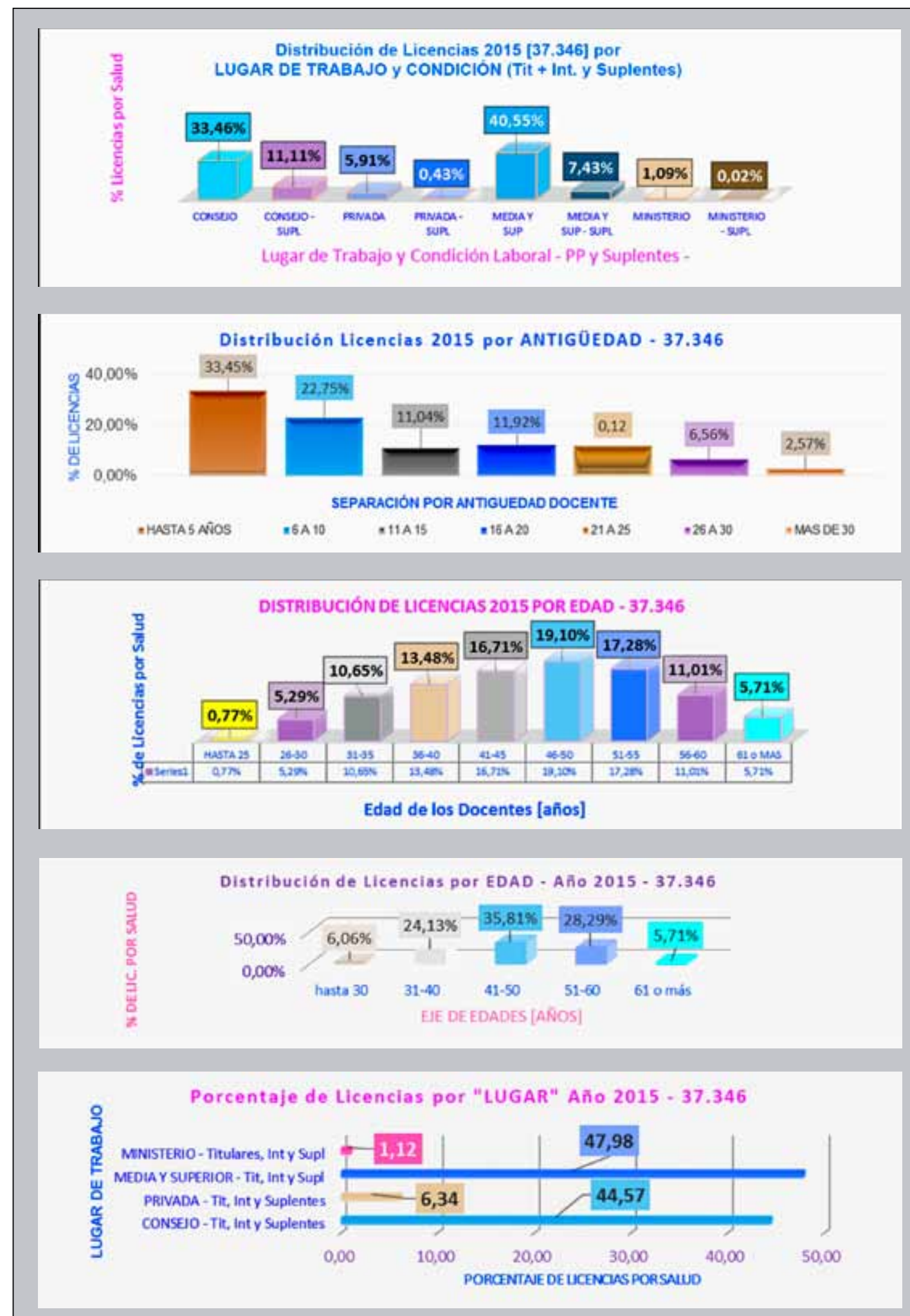

Gráfico No 6: Representación de los Cuadros Expuestos - Licencias de Salud 2015 


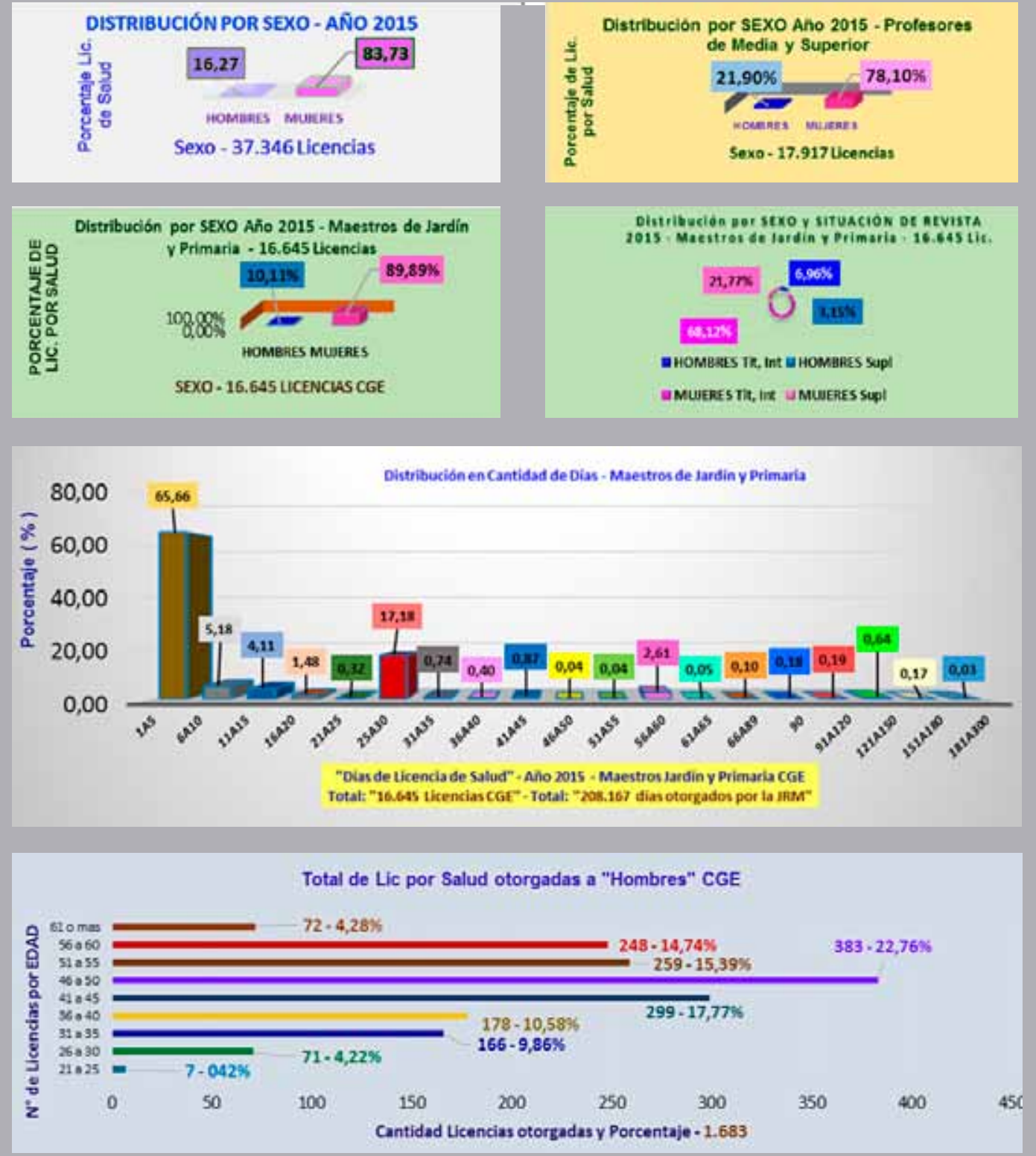

Total de Lic por Salud otorgadas a "Mujeres" CGE

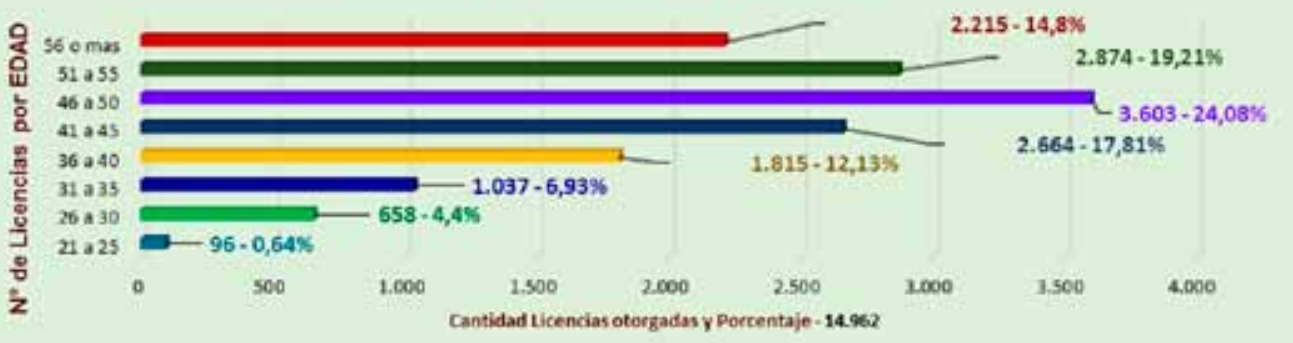

Gráfico No 7: Representación de los Cuadros JRM - Sexo, Dias otorgados y Edades Licencias 2015 
- DIMENSION PATOLOGIAS

Segmentación de las Patologías:
Se procede a la segmentación del Cuadro $\mathrm{N}^{\circ}$ 11, en 21 Patologías tipificadas por la JRM

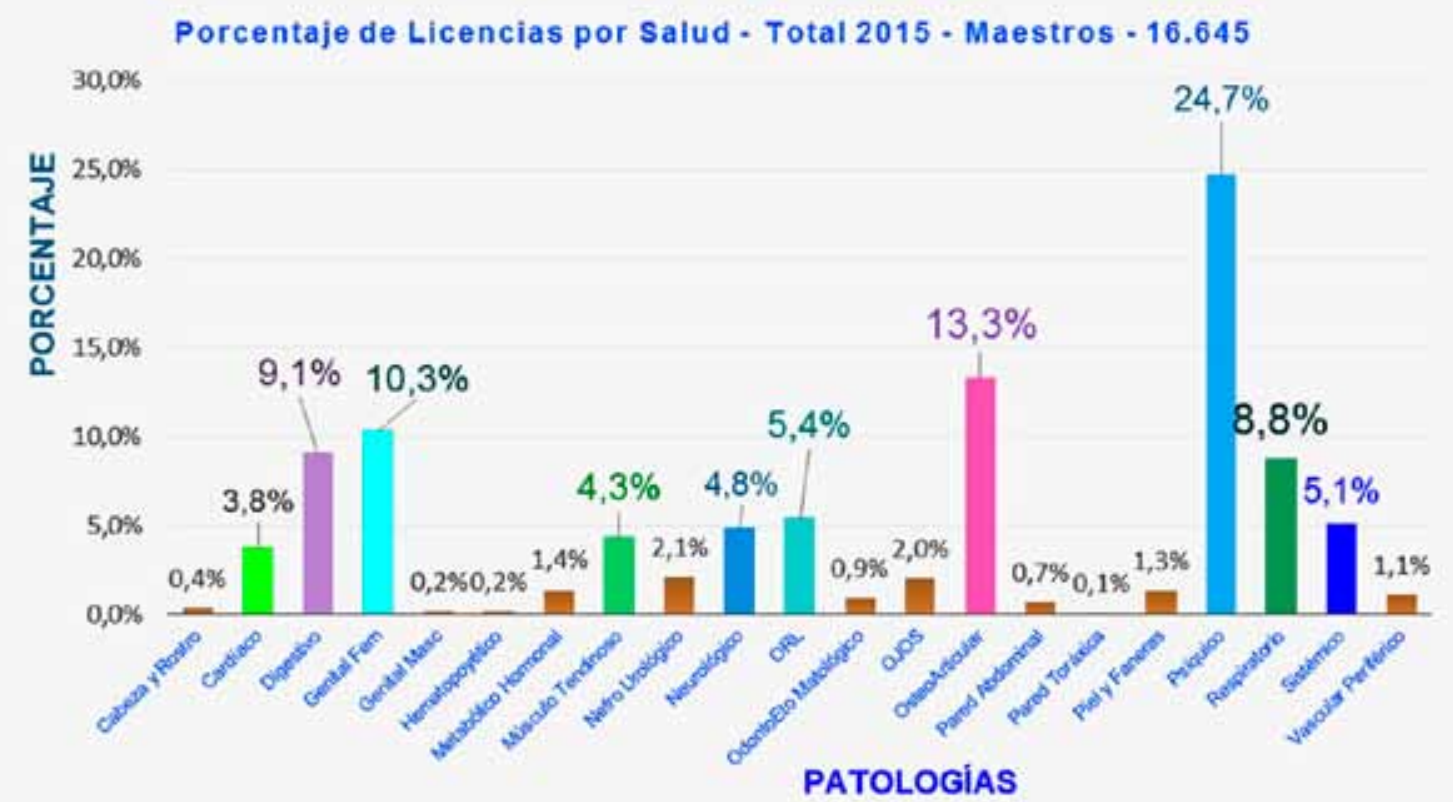

Gráfico No 8: Licencias de Salud de Trabajadores Docentes de Nivel Inicial y

Primario JRM 2015

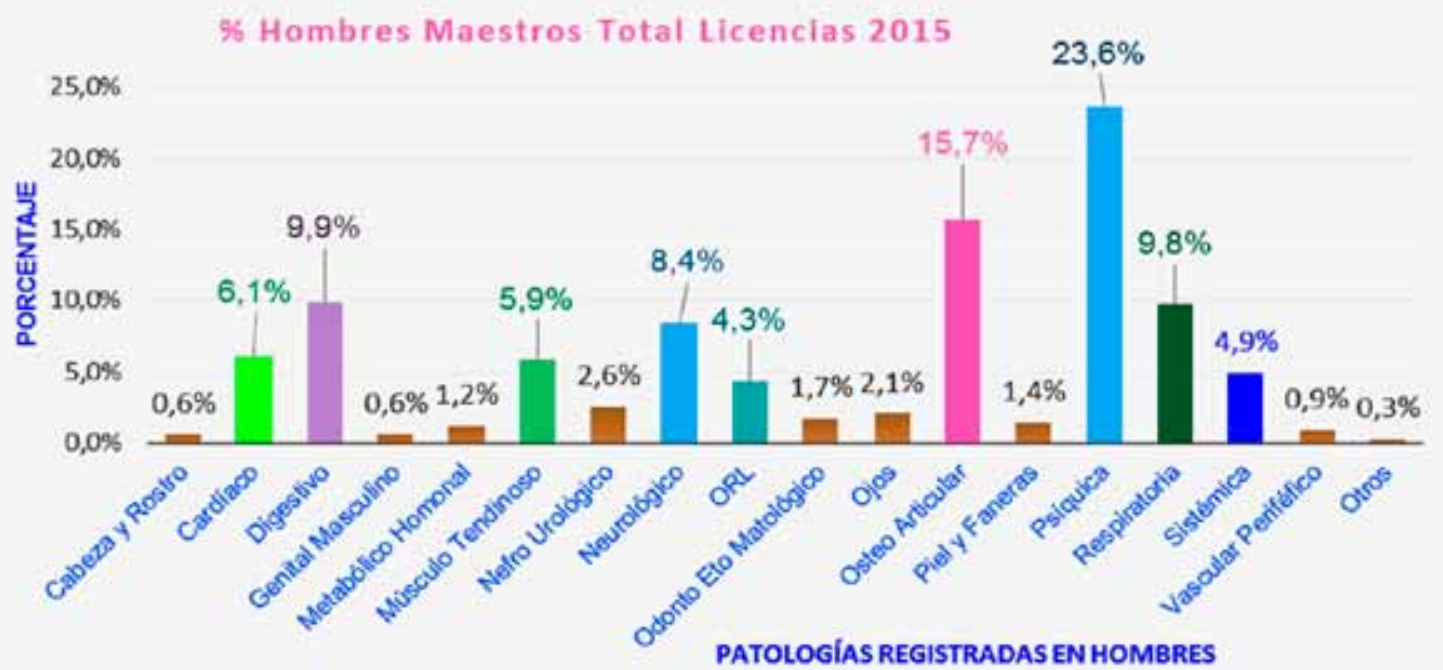

Gráfico No 9: Licencias de Salud de Docentes Hombres de Nivel Inicial y Primario JRM 2015 


\section{\% Mujeres Maestras Total Licencias 2015}

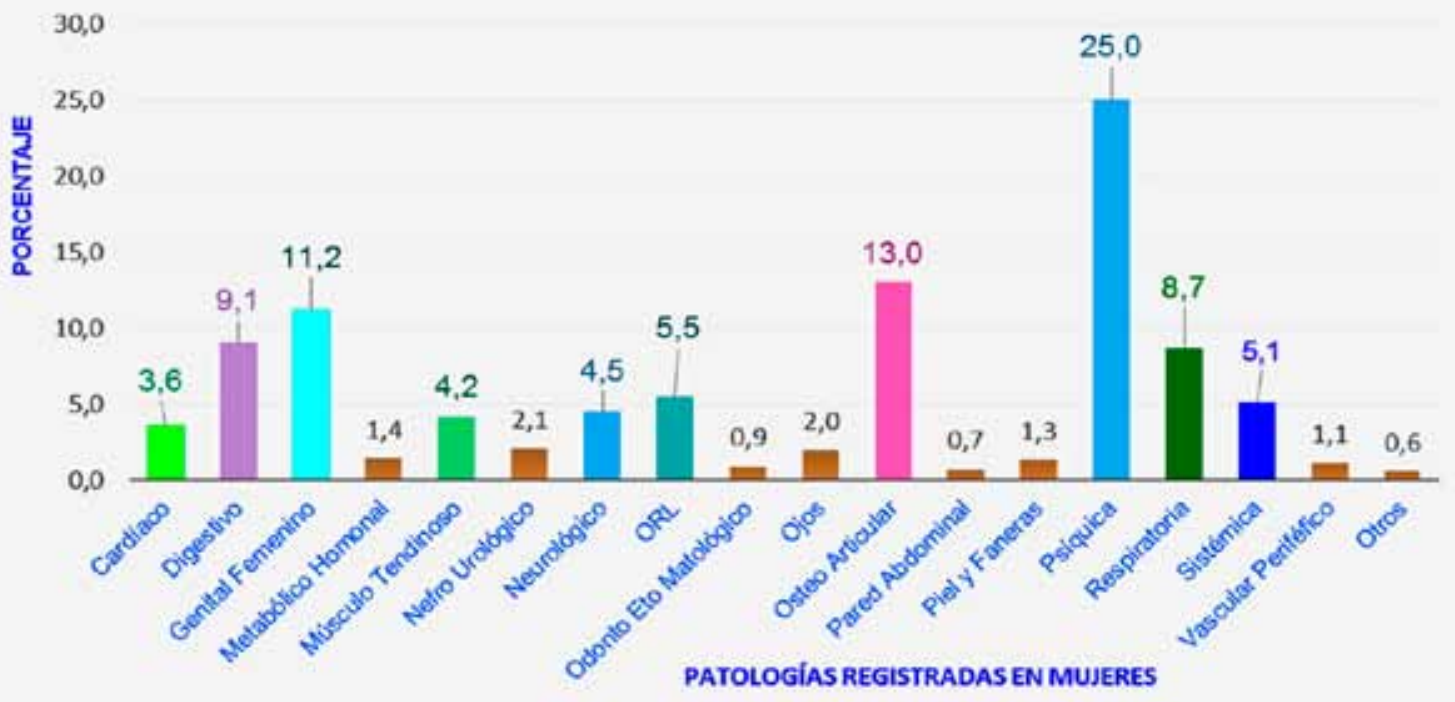

Gráfico No 10: Licencias de Salud de Docentes Mujeres de Nivel Inicial y Primario JRM 2015

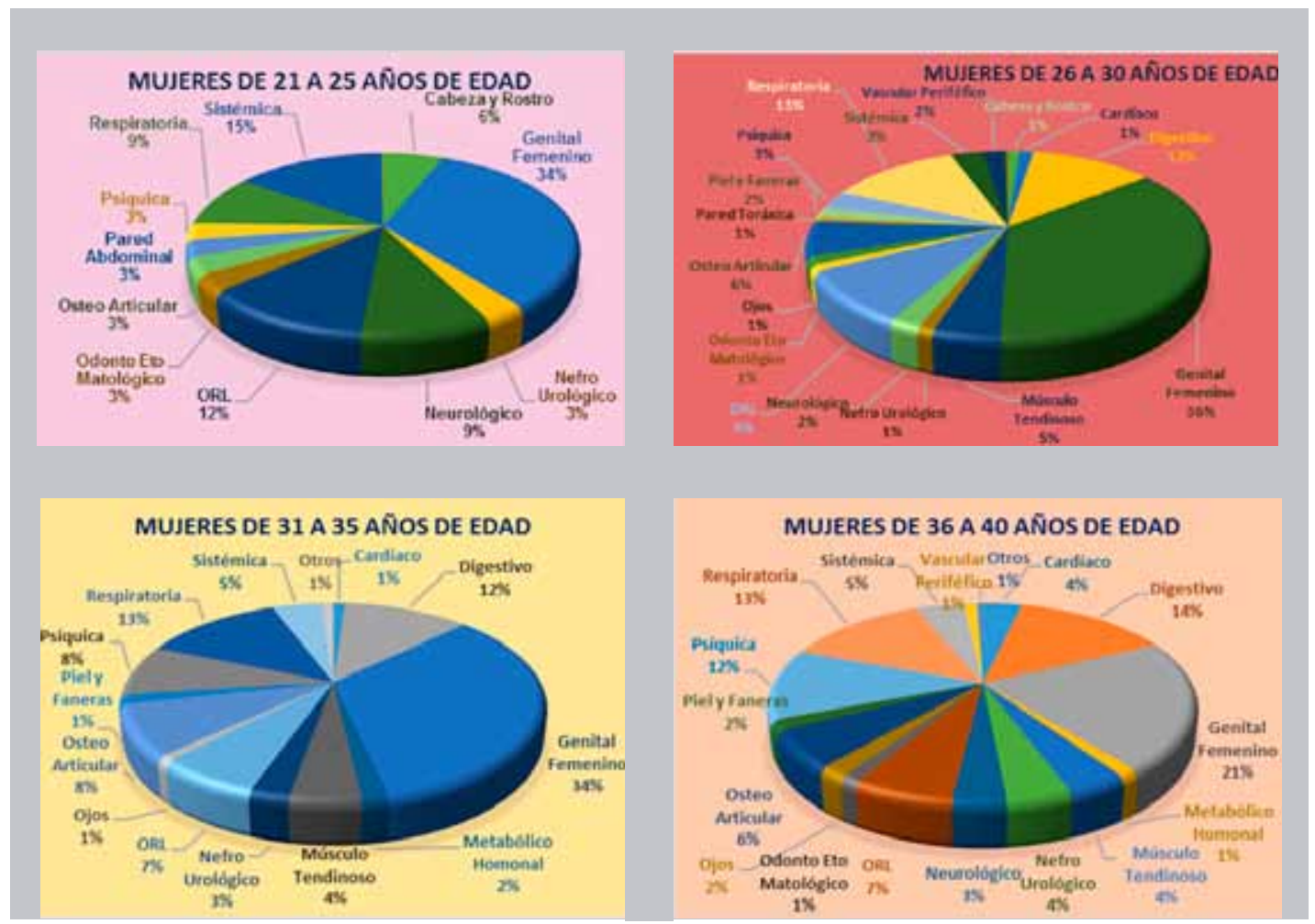



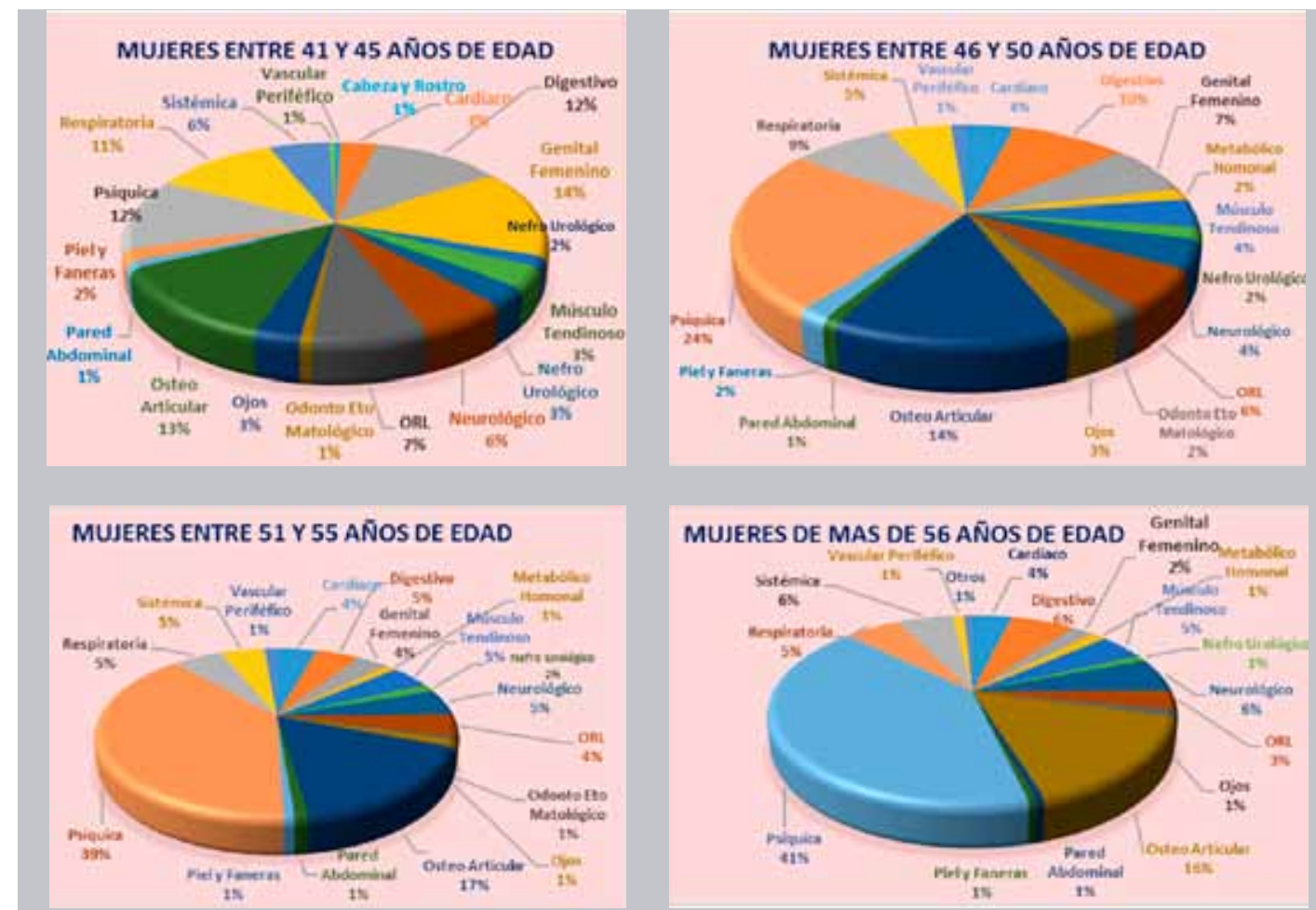

Gráfico No 11: Patologías de Mujeres por EDAD de Nivel Inicial y Primario JRM 2015 

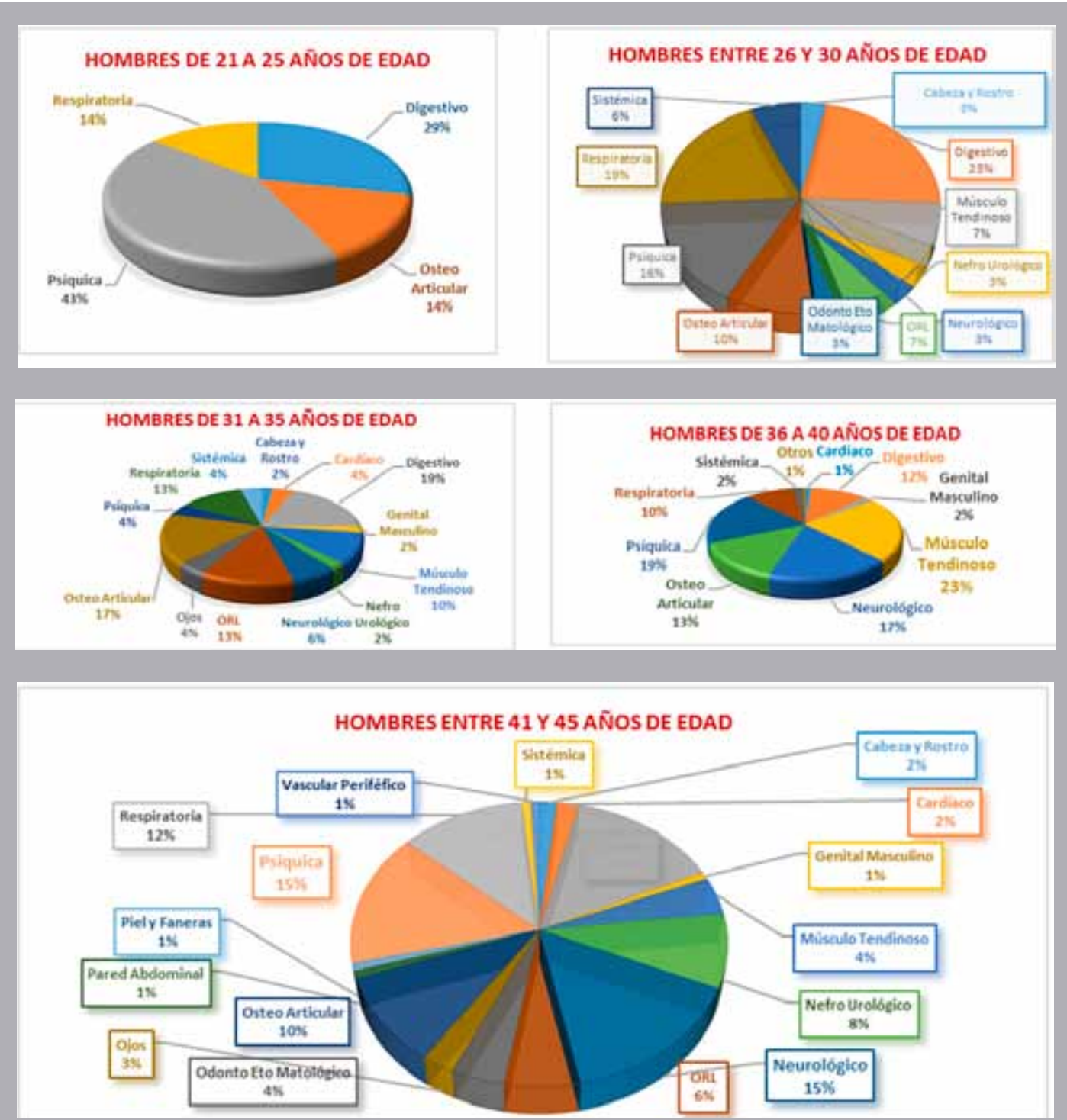

\section{HOMBRES ENTRE 46 Y 50 AÑOS DE EDAD: 117 CASOS}

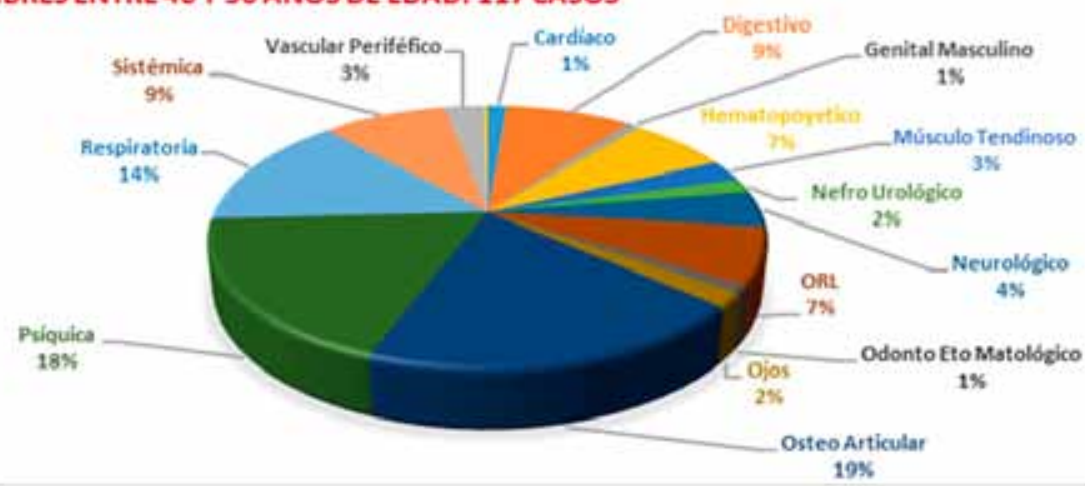

Gráfico No 12: Patologias de Hombres por EDAD Maestros hasta 50 años de edad - 


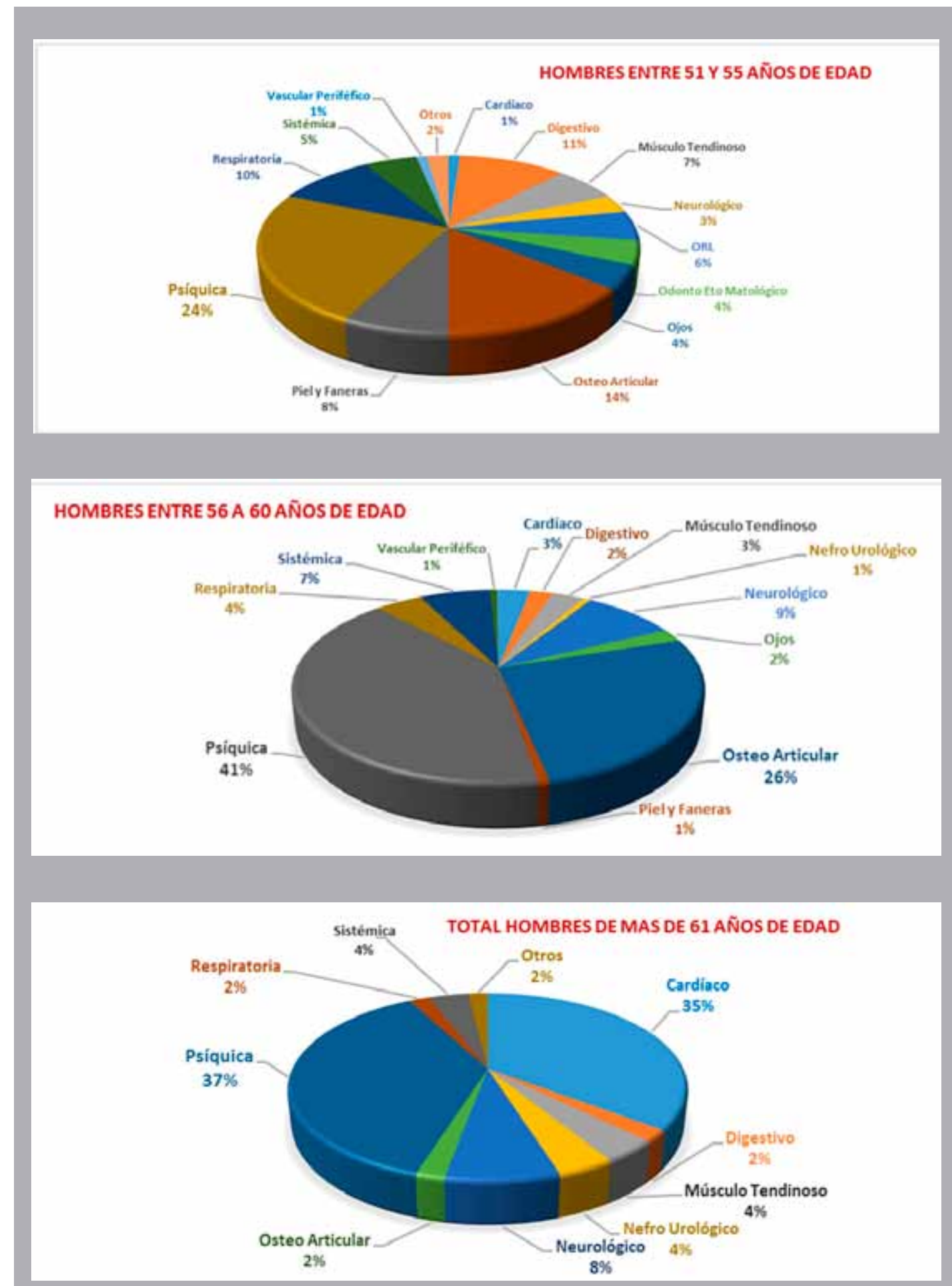

Gráfico No 13: Patologías de Hombres por EDAD

Maestros de Más de 51 años de edad-JRM 2015 

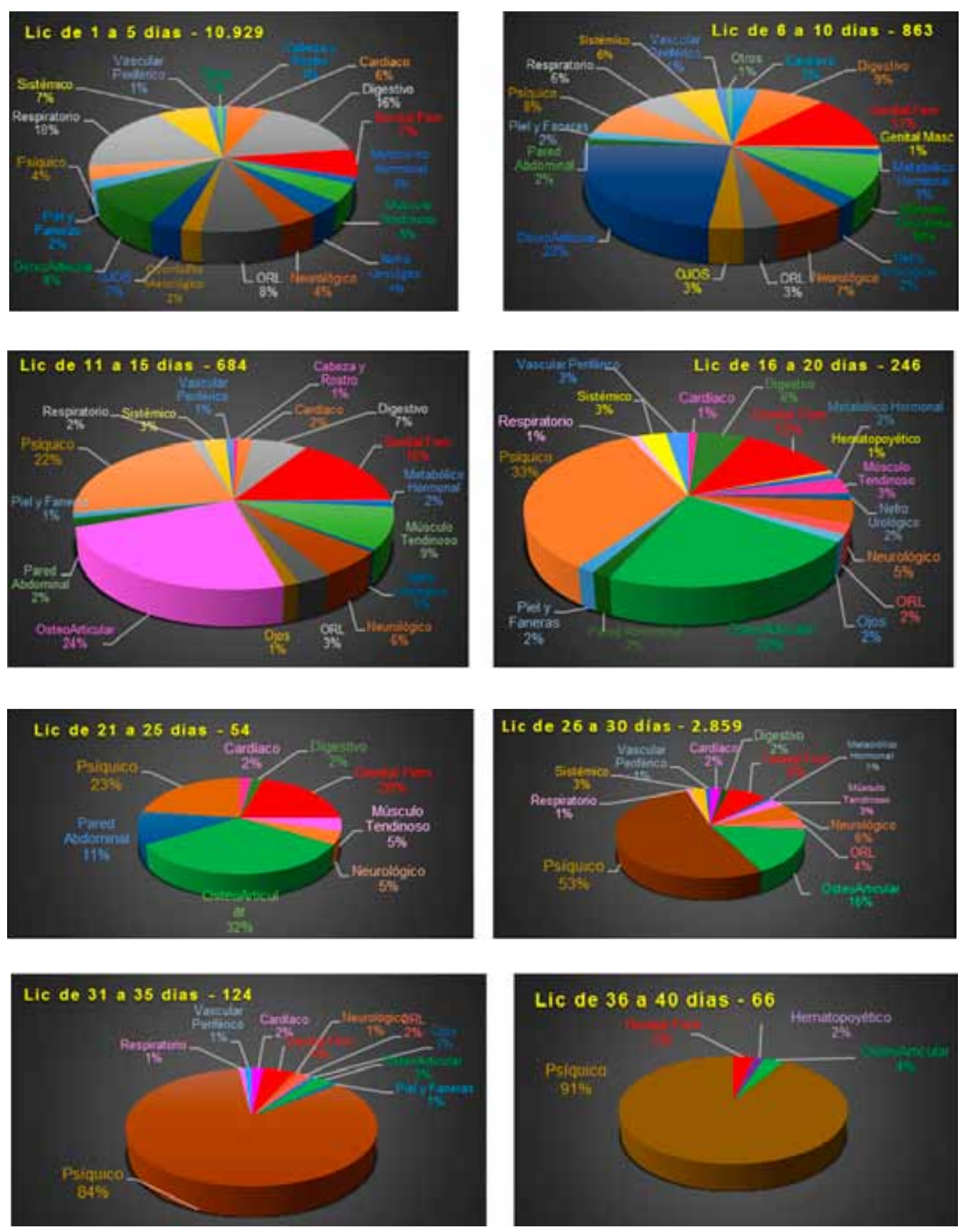

Gráfico No 14: Patologías segmentadas por Cantidad de DÍAS de Licencia otorgados, de 1 a 40 dias 

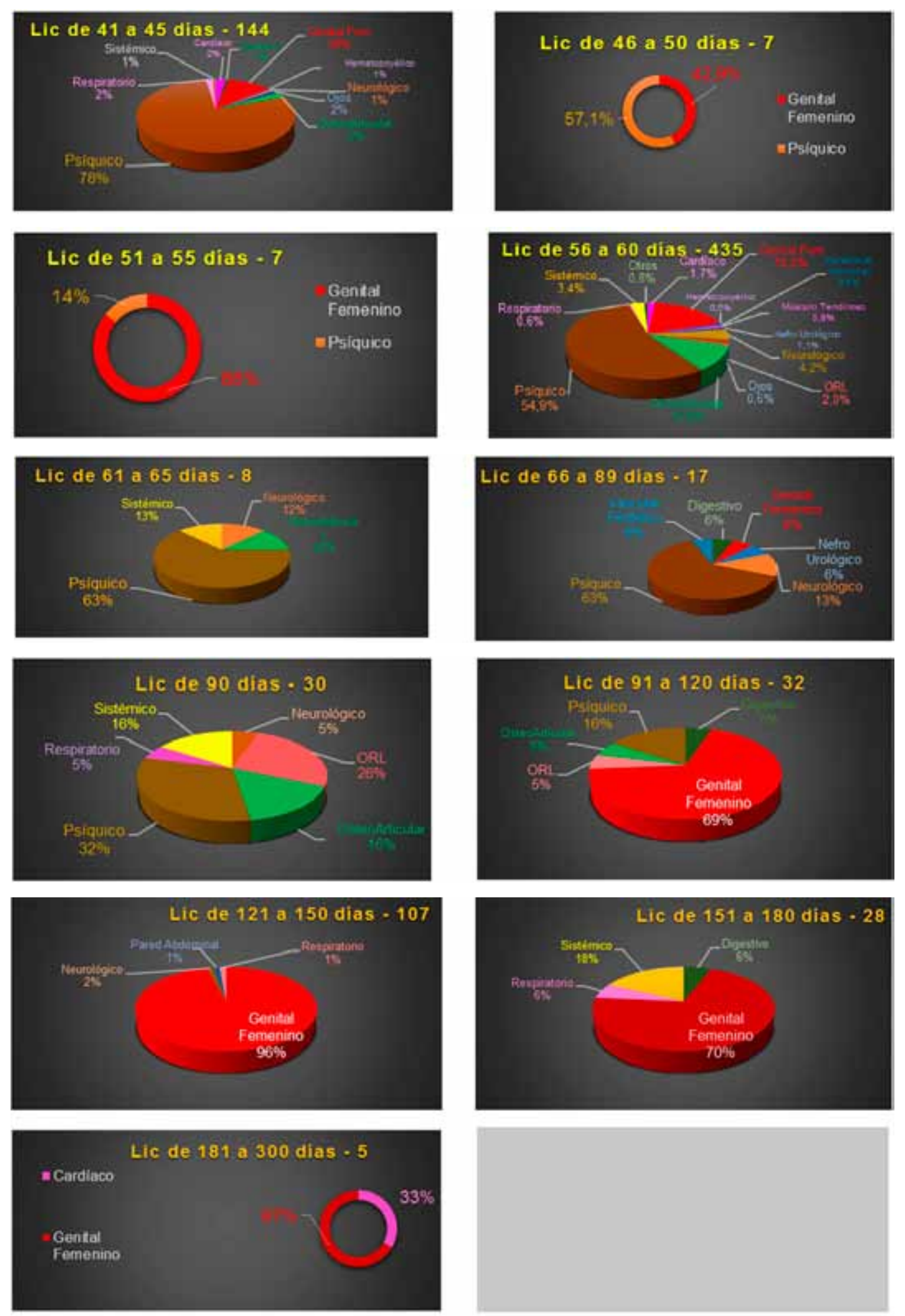

Gráfico No 15: Patologías segmentadas por Cantidad de DÍAS de Licencia otorgados de 41 a 300 días 
VARIABLE ESTADO DE SALUD

DE LOS DOCENTES: SUBDIMENSION MEDICAMENTOS

Los 32 medicamentos más prescriptos y autorizados por la Obra Social IOSCOR:

\begin{tabular}{|c|c|c|}
\hline $\mathbf{N}^{0}$ & MEDICAMENTO & DROGA \\
\hline 1 & ENSURE PLUS DRINK & PROTEINAS+GRASAS+CARBOHID + +ASOC. \\
\hline 2 & LOTRIAL & ENALAPRIL \\
\hline 3 & CARVEDIL & CARVEDILOL \\
\hline 4 & CLONAGIN & CLONAZEPAM \\
\hline 5 & LOSACOR & LOSARTAN \\
\hline 6 & ALPLAX & ALPRAZOLAM \\
\hline 7 & $\begin{array}{l}\text { ACCU-CHEK PERFORMA } \\
25\end{array}$ & TIRAS REACTIVAS \\
\hline 8 & DBI AP METFORMINA & METFORMINA \\
\hline 9 & $\begin{array}{l}\text { ACCU-CHEK ACTIVE } \\
\text { GLUCOSA }\end{array}$ & TIRAS REACTIVAS \\
\hline 10 & OPTAMOX DUO & AMOXICILINA+CLAVULANICO AC. \\
\hline 11 & $\begin{array}{l}\text { ACTRON } 600 \text { RAPIDA } \\
\text { ACCION }\end{array}$ & IBUPROFENO \\
\hline 12 & ATENOLOL GADOR & ATENOLOL \\
\hline 13 & DIUREX & HIDROCLOROTIAZIDA \\
\hline 14 & GLIOTEN & ENALAPRIL \\
\hline 15 & $\begin{array}{l}\text { ACCU-CHEK PERFORMA } \\
50\end{array}$ & TIRAS REACTIVAS \\
\hline 16 & DIOVAN & VALSARTAN \\
\hline 17 & RIVOTRIL & CLONAZEPAM \\
\hline 18 & AMPLIAR & ATORVASTATIN \\
\hline 19 & FILTEN & CARVEDILOL \\
\hline 20 & PAXON & LOSARTAN \\
\hline 21 & TERLOC & AMLODIPINA \\
\hline 22 & PELMEEC & AMLODIPINA \\
\hline 23 & CORTEROID & BETAMETASONA \\
\hline 24 & MACRIL & BETAMETASONA+GENTAMIC+MICONAZOL \\
\hline 25 & AMOXIDAL & AMOXICILINA \\
\hline 26 & ULCOZOL & OMEPRAZOL \\
\hline 27 & LIPITOR & ATORVASTATȦN \\
\hline 28 & SIDERBLUT & HIERRO, SULFATO \\
\hline 29 & ISTIVAC & ANTAGENOS VIRUS INFLUENZA \\
\hline 30 & T4 MONTPELLIER 100 & LEVOTIROXINA SODICA \\
\hline 31 & NOVALGINA & DIPIRONA \\
\hline 32 & CORTEROID RETARD & BETAMETASONA (ACET.Y FOSF.DISOD) \\
\hline
\end{tabular}

Cuadro $N^{\circ}$ 6: Medicamentos con mayor número de prescripciones en la Obra Social - Año 2015 
CONCLUSIONES Y PROPUESTAS A PARTIR DEL ESTUDIO DE LA PROBLEMÁTICA

Para el presente Trabajo se estudiaron la totalidad de las Licencias de Salud otorgadas y registradas en 21 Patologías por la JRM de Corrientes y el listado de los 32 Medicamentos autorizados en mayor número por la Obra Social IOSCOR, durante un año.

A partir del procesamiento (segmentación y representación gráfica) y el análisis de los mismos, incluyendo los conceptos vertidos por los IC, se consideran más relevantes para el grupo objeto de estudio (maestros de grado común de niveles Inicial y Primario de escuelas públicas de la provincia de Corrientes dependientes del CGE).

Relacionando las conclusiones y análisis con lo propuesto por Neffa (1988 y 2015), quien sostiene que los trabajadores deben ser reconocidos como seres individuales y no como sujetos homogéneos y que, además, el proceso de trabajo comienza a tomarse en su aspecto micro y macro, entendiendo que las CyMAT están relacionadas con las condiciones que hacen a la carga física, a la seguridad en él y a la carga psíquica y mental del trabajador.Se puede concluir afirmando quesin haber analizado en profundidad todas las variables de las condiciones y medio ambiente de trabajo en la que desarrollan su labor los docentes del nivel inicial y primario, las dimensiones de la salud afectada varían de acuerdo a la edad y sexo de los docentes. En el caso de las mujeres la dimensión de la saludfísica es afectada desde los primeros años hasta los 45 años, a partir de esta edad surgen y hasta su egreso del sistema laboral,la dimensión mayor afectada es la psíquica.
En el caso de hombre los primeros años son afectados por la dimensión psíquicos, a partir de los 30 y hasta 50 años se equiparan los problemas en la dimensión física y psíquica y mental. A partir de los 50 años vuelven a aumentar en forma creciente los problemas de la demisión psíquica, hasta su egreso del sistema laboral.

Habiendo analizado en forma sistematizada cada una de las variables y respondidos los interrogantes del presente estudio se enumerarán algunas propuestas al Estado en función del presente trabajo que podrían favorecer las condiciones del trabajo docente, reduciendo, en el caso de ser satisfactorias, el número de licencias por enfermedad y disminuir el costo oculto que esto ocasiona.

- Diseñar un Sistema de Descentralización para la Atención y Prevención de Enfermedades exclusivo para Trabajadores en ejercicio de la Docencia. Estos podrían darse con la creación de Centros de Salud por Regiones, existiendo la posibilidad de instaurar oficinas / consultorios itinerantes, estableciéndose fechas predeterminadas para cada Unidad Educativa. Los Centros tendrían entre sus funciones atender y registrar el estado de salud de los docentes, las visitas a los establecimientos del personal de salud, remitir consultas médicas frecuentes de los docentes a las áreas correspondientes (Unidades Sanitarias Especializadas), brindar capacitación sobre las patologías según la región, temporada, característica socio-económica de los alumnos, el rango de edad y sexo del trabajador. El objeto fundamental de esta propuesta es prevenir aquellas enfermedades que están relacionadas con el Puesto de Trabajo y que motiva la solicitud de Licencia Docente para el tratamiento de la Salud. 
Con el registro digitalizado se posibilitaría crear una Base Única de Datos de Enfermedades, Capacitaciones a docentes $\mathrm{y}$ funcionarios $\mathrm{y}$ Registro de atenciones médicas preventivas a Docentes en el lugar de trabajo, poniendo a disposición de las autoridades nuevos indicadores para la toma de decisiones.

- Incorporar un Portal de Servicios Interactivo y personalizado para los mismos, al sólo efecto de recibir demandas/opiniones y brindar información sobre cuestiones relacionadas a las Condiciones y el Medio Ambiente de Trabajo: diseño curricular, relación con superiores, colegas y alumnado, remuneraciones, servicios sociales, procedimientos administrativos en general para agilizarlos, equipamiento e infraestructura, entre otros. Esto permitiría una comunicación más fluida entre docentes y otros miembros de la Organización, motivándolos a participar de las decisiones que los involucren directamente en su labor diaria y les dé un sentido de pertenencia en el Establecimiento Educativo. "E1 grado de aceptación o de cuestionamiento a las CyMAT y los RPST que se puede conocer realizando entrevistas a informantes calificados, recibiendo quejas y observaciones de los trabajadores y analizando en las actas el desarrollo de las reuniones de los comités mixtos de salud, seguridad y condiciones de trabajo" Neffa (2015).

- En general en el Ministerio de Educación los procesos administrativos son lentos y no homogéneos, con estructuras fuertemente jerarquizadas y centralizadas, lo quedificulta la comunicación interna y externa, subutilizando las TIC y elevando costos operativos. Las acciones tienden a ser correctivas más que preventivas. Para éste problema se propone implementar un
Sistema Telemédico (TM), donde agentes sanitarios dependientes de cada Centro Regional, mediante móviles preparados al efecto para recorrer las Escuelas, puedan obtener datos mínimos pero esenciales de la Salud del trabajador, como Monitor Multiparamétrico de registro digital de bioseñales: Electrocardiogramas ECG, Electroencefalogramas EEG, Presión Arterial No Invasiva BPNI y Saturación de Oxígeno de Sangre; Termómetro digital; Estetoscopio electrónico de grabación de las auscultaciones y filtro de ruido ambiental; Balanza; Monitor digital de Presión en Sangre; Cámara Digital de Alta Resolución HD; Notebook con conectividad de alta performance y Ficha Médica Digital.

La telemedicina (TM) busca la atención clínica de pacientes, mediante la utilización de medios remotos y el aprovechamiento de las TIC.La práctica a distancia de la Medicina o TM, incluye el diagnóstico y el tratamiento remoto, y es un recurso que posibilita la optimización de los servicios de atención de la salud, facilitando el acceso a zonas distantes la atención de especialistas.

Con ello se reducen de manera sustancial los costos de tiempo y dinero, permitiendo no sólo el acceso remoto a los pacientes docentes, sino también la derivación oportuna a los centros de salud regional para la intervención adecuada del profesional de la salud. Dentro de esta modalidad de práctica médica se puede capacitar sobre prevención de enfermedades, la telediagnósis (primer diagnóstico mediante el uso de las telecomunicaciones), la teleconsulta, el monitoreo remoto, la teleconferencia entre médicos para segundas opiniones y el almacenamiento y seguimiento de las fichas médicas.TM permitiría la comunicación en tiempo real con agentes en distintos puntos geográficos. 
A pesar de la aparente complejidad de éste Servicio, simplemente se pueden usar los recursos disponibles gratuitos para la comunicación web audiovisual como Teamviewer o Skipe. La aparatología descripta no es muy onerosa y el adiestramiento de los agentes sanitarios para manipular ésta tecnología es relativamente sencillo. Se puede complementar con la extracción de muestras de sangre y el posterior análisis bioquímico.

- Implementar políticas que permitan identificar y "prevenir" los RPST de los docentes, atendiendo la Región y las condiciones en la cual desarrolla su trabajo. $\mathrm{Si}$ vemos las observaciones realizadas por los Informantes Claves más algunos resultados del TFI, la inversión en dinero a largo plazo puede verse reducida, a par-

\section{BIBLIOGRAFÍA}

1 Fugliestaler, Graciela (2010), Tesis "Importancia del nivel educativo y la antigüedad en el puesto de los empleados para la identificación de los factores intervinientes en la satisfacción laboral en una organización pública" Carrera Lic. Relaciones Laborales UNNE.

2 García Fariña, Mariángeles (2010) tesis "Condiciones de trabajo docente y factores de riesgos en establecimientos escolares a nivel primario del Departamento de Mercedes de la Provincia de Corrientes" Carrera Lic. Relaciones Laborales UNNE tir de la disminución del gasto en salarios durante dure la enfermedad del docente, de la cobertura de gastos en consultas médicas, fármacos y estudios, del gasto oculto con el pago de nuevos sueldos por suplencias, considerando más importante la instrumentación de políticas para mejorar la calidad de vida del trabajador docente.

- Finalmente se propone tener en cuenta las edades analizadas en el marco teórico y sus características descriptas en Generaciones, para una implementación exitosa de los Sistemas y las Políticas consideradas precedentemente. Cabe señalar que todo proceso de cambio en una Organización genera resistencia, por parte de sus integrantes: funcionarios, docentes, otros trabajadores del sistema.

3. González Trigueros, L.; Hernando Saudán, A. et al (2003) "Dorland Diccionario Enciclopédico Ilustrado de Medicina” ISBN 84-486-0317-6 Editorial Mc Graw Hill.

4. Kandel, E; Schwartz, J; Jessell, T (2003) "Neurociencia y Conducta" ISBN 8489660-05-0 Editorial Prentice Hall.

5. Mandell, G.; Bennett, J.; Dolin, R. (2004) "Enfermedades Infecciosas, Principios y Práctica” V Edición ISBN 950-061570-3 Editorial Médica Panamericana. 
6 Martínez, Deolidia (1990) El Riesgo de Enseñar. Secretaria de Cultura Y Educación. Buenos Aires.

7. Maslach, Christina; Schaufeli, Wilmar; Leiter, Michael (2001). "Job Burnout”. Annual Review of Psychology 52 (1): 397-422. ISSN 0066-4308.

8 Montero-Marín, Jesús; García-Campayo, Javier (2010). "A newer and broader definition of burnout: Validation of the Burnout Clinical Subtype Questionnaire (BCSQ-36)". BMC Public Health (10): 302. ISSN 1471-2458.PMID 20525178

9 Neffa, Julio Cesar (1988) ¿Qué son las condiciones y Medios ambiente de Trabajo? Propuesta de una nueva Perspectiva, Cap IV. CREDAL- Unidad asociada No 111 al Center Nacional de la RechercheScientifique (CNRS) Editorial Hvmanitas.

10. Neffa,Julio Cesar(2015)Los Riesgos Psicosociales en el Trabajo. Contribución a su estudio. Libro Digital ISBN 978-987-21579-9-9.

11 Putz, R.; Pabst, R.(2003) “Atlas de Anatomía Humana Sobotta”XXIEdición ISBN3-43741940-4 Editorial Médica Panamericana.

12 Ross, Sheldon (2002) "Probabilidad y Estadística para Ingeniería y Ciencias” ISBN 970-10-3456-2 Editorial Mc Graw Hill.

13 Sabino, Carlos (1996) El proceso de Investigación- Editorial Humanitas, Bs As.

14.Schaufeli, Wilmar B.; Bakker, Arnold B.; Schaap, Cas; Hoogduin, Kees; Kladler, Atilla (2001) "On the clinical validity of the Maslach Burnout Inventory and the Burnout Measure". Psychology \& Health 16 (5): 565-582. ISSN 0887-0446.

15.Simbula, Silvia; Guglielmi, Dina (2010). "Depersonalization or cynicism, efficacy or inefficacy: what are the dimensions of teacher burnout?" European Journal of Psychology of Edu- cation 25 (3): 301-314. ISSN 0256-2928.

16 Thibodeau, G.; Patton, K. (1999) "Anatomía y Fisiología” IV Edición ISBN 84-8174-449-2 Editorial Diorki.

17 Torres, María Rosa (2000) De agentes de la reforma a sujetos del cambio: la encrucijada docente en América Latina. Perspectivas, $\mathrm{XXX}(2)$

18 Verón, O.E. (2003) Lineamientos Estratégicos. Superintendencia de Riesgos de Trabajo. Ed. SRT. Bs As.

\section{NORMATIVAS}

$>$ Decretos de la Provincia de Corrientes No $1.482 / 79$ y No $1.629 / 79$.

$>$ Ley No 3.723 (Estatuto del Empelado Docente de la Provincia de Corrientes).

> Ley N 26.206 (Ley de Educación Nacional capitulo II Artículo 20\%).

\section{BIBLIOGRAFIA DE INTERNET}

$>$ http://www.eumed.net/rev/ced/02/cam6. htm

$>$ h t t p : / / d e fin i c i o n.d e / salud/\#ixzz40ZTuTHRR

$>$ http://www.clinicaerasmo.com/index. $\mathrm{php/component/content/article/38-salud/}$ salud/94-trabajosalud

$>$ http://www.tonetunes.net/tunes/americanburnout

$>$ http://www.tonetunes.net/tunes/americanburnout

$>$ http://revistasuma.es/IMG/pdf/45/017028.pdf

http://es.slideshare.net/cobos288/generaciones-32405249

> http://www.slideshare.net/hansmejia/diseocurricular-36517973 